\title{
A Fecalith Mimicking a Bladder Calculus Secondary to an Appendicovesical Fistula
}

\author{
Mauricio Gonzalez-Urquijo ${ }^{1,2}$, Andrea Romero-Davila ${ }^{1}$, MaryCarmen Mendoza-Silva ${ }^{1,2}$, \\ Antonio Nassim Halun Treviño, ${ }^{1,2}$, Mario Rodarte-Shade ${ }^{1,2}$, Gerardo Gil-Galindo ${ }^{1,2}$ \\ ${ }^{1}$ Department of Surgery, Tecnologico de Monterrey, Escuela de Medicina y Ciencias de la Salud, Monterrey; ${ }^{2}$ Department of Surgery, Hospital \\ Metropolitano "Dr. Bernando Sepúlveda”, San Nicolás de los Garza, México
}

An appendicovesical fistula is defined as an abnormal communication between the appendix and the urinary bladder; with only a few cases reported in the literature. It is very challenging to make an early diagnosis, due to the inability of conventional imaging modalities to detect this unique pathology. The symptoms are often mild, and there are not any specific signs or symptoms that might suggest this type of anomalous communication. We report a case of a 27 -year-old male patient who presented difficulty for initiating urination, dysuria, and persistent urinary tract infections. An abdominal X-ray showed a large calculus inside the bladder. A cystoscopy was performed, where the tip of the appendix was seen protruding inside the bladder with a large fecalith adhered to the bladder wall. An appendectomy and partial cystectomy with primary repair were auspiciously achieved. A review of the literature is also presented.

Keywords: Urinary bladder fistula; Fecal impaction; Appendix; Appendicitis

\section{INTRODUCTION}

An appendicovesical fistula (AVF) was first described by Keen in 1898 [1] and can affect both adults and children [2], representing approximately $1 \%$ to $5 \%$ of all enterovesical fistulas [3, 4]. An AVF is defined as an unual communication between the appendix and the urinary bladder, because of the inflammation or malignancy and rupture of the first inside the second [5]. It has been seen following diverse diseases including acute appendicitis, Crohn disease, radiation enteritis, papillovillous adenoma of the appendix, appendicular diverticulitis, cystadenocarcinoma of the appendix, carcinoid tumors, cystic fibrosis, and Hirschsprung disease $[4,6-8]$. It is very challenging to make an early diagnosis, due

Received: Apr 20, 2020 - Revised: Jun 17, 2020 - Accepted: May 16, 2021 Correspondence to: Mauricio Gonzalez-Urquijo, M.D.

Department of Surgery, Tecnologico de Monterrey, Escuela de Medicina y Ciencias de la Salud, Dr. Ignacio Morones Prieto 0 3000, Monterrey 64710, México

Tel: +52-8119103675

E-mail: mauriciogzzu@gmail.com

ORCID: https://orcid.org/0000-0001-5101-1541

(c) 2021 The Korean Society of Coloproctology

This is an open-access article distributed under the terms of the Creative Commons Attribution NonCommercial License (https://creativecommons.org/licenses/by-nc/4.0) which permits unrestricted noncommercial use, distribution, and reproduction in any medium, provided the original work is properly cited. to the inability of conventional imaging modalities to detect this unique pathology. The symptoms are often mild, and there are not any specific signs or symptoms that might suggest this type of anomalous communication [6]. Usually, the duration between the initial event and the final diagnosis is reported to be long, up to many years [2].

Since 2006, 116 cases of AVF have been described; approximately $10 \%$ to $15 \%$ of them were children [2]. Herein we report a case of a 27-year-old male patient who presented with urologic symptoms, and a final diagnosis of an AVF was made. A review of the literature of this rare entity is made.

\section{CASE REPORT}

A 27-year-old man was admitted to the emergency department referring difficulty for initiating urination, dysuria, and he referred persistent urinary tract infections (UTIs) associated with mild recurrent periumbilical and lower abdominal pain. On physical examination, abdominal tenderness at right lower quadrant was noted. Routine blood counts and biochemical analyses were within normal parameters, and an abdominal X-ray was ordered, where a large calculus was seen on the bladder (Fig. 1). Urinalysis showed more than 50 white blood cells per high power field (HPF), many bacteria per HPF, and positive nitrites, and on 


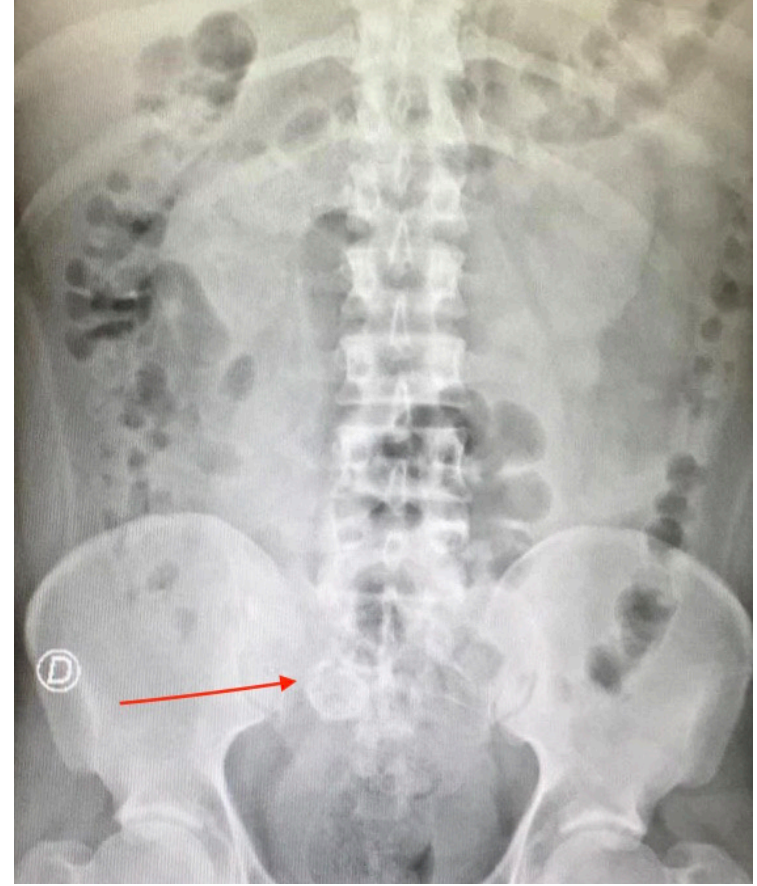

Fig. 1. Abdominal X-ray with a large calculus inside the bladder (red arrow).

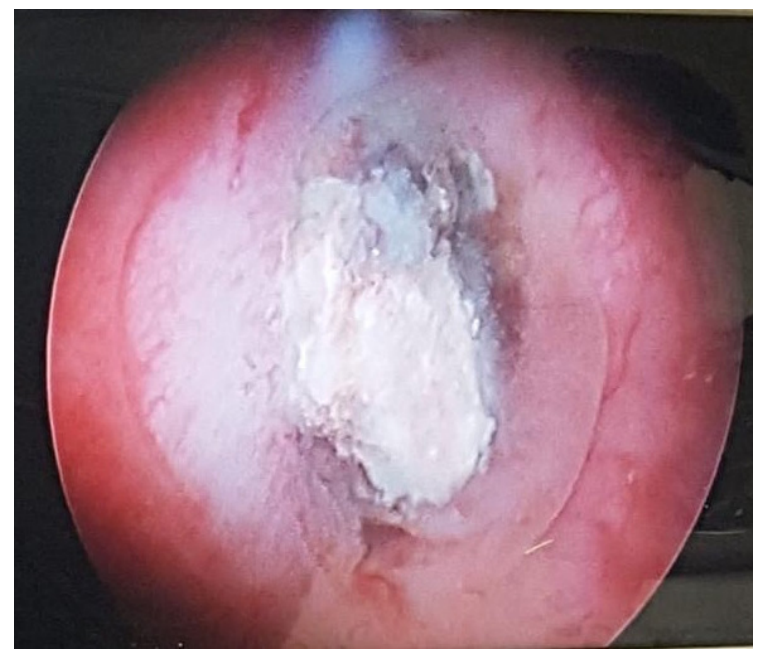

Fig. 2. Cystoscopy showing the appendicular tip protruding through the bladder cavity.

the urine culture test Escherichia coli was obtained.

Because the patient was in a lot of pain, without any improvement after receiving multiple doses of analgesic, the patient was transferred to the operating room, where a cystoscopy was performed, observing a $4-\mathrm{cm}$ fecalith on the right lateral wall of the bladder dome, which was completely adhered to the bladder's wall (Fig. 2). So the decision to perform a midline incision was

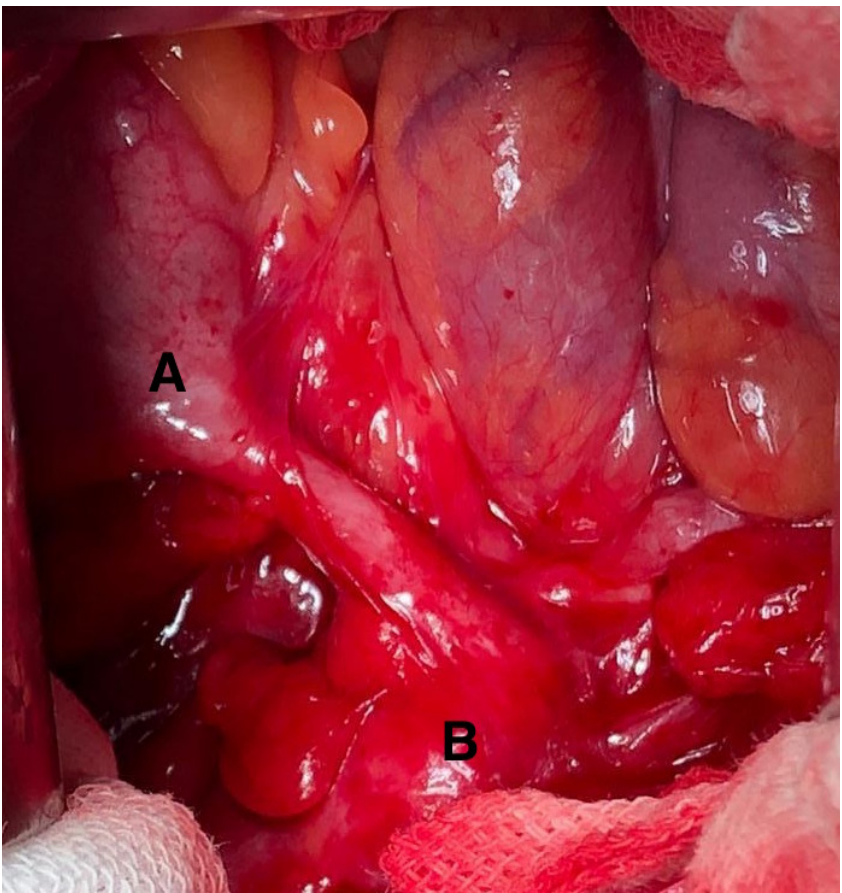

Fig. 3. Anomalous communication between the appendix (A) and the bladder (B).

made. The tip of the appendix was firmly adhered and eroded into the cavity of the bladder (Fig. 3). A partial cystectomy was performed, and while the tip of the appendix was being removed, the $4-\mathrm{cm}$ fecalith was seen. The fistulous tract was resected, and the appendectomy was performed without complications. Finally, the bladder was repaired in 2 layers. A urine catheter was placed and was removed on postoperative day (POD) 14. The patient was discharged on POD 5 with oral antibiotics, and with clear urine. Histological examination of the appendix showed acute and chronic inflammatory changes with ulceration of the mucosa and transmural fibrosis.

Written informed consent was obtained from the patient for publication of this case report and accompanying images. All procedures performed in studies involving human participants were in accordance with the ethical standards of Tecnologico de Monterrey Ethics Committee and have therefore been performed in accordance with the ethical standards laid down in the 1964 Declaration of Helsinki and its later amendments.

\section{DISCUSSION}

The creation of a fistula, which follows the rupture and drainage of the cecal appendix inside the bladder cavity is rare. Achieve the diagnosis of an AVF is more difficult than any other enterovesical fistula along the gastrointestinal tract because the latter often gives signs of pneumaturia or fecaluria. Different from the former, where the lumen of the appendix is smaller, and the length of the 
track is much greater than their enterovesical counterparts, so a periodic complete obstruction may be caused by a calculus or fecalith [5]. In our case, the first sign we saw was a large fecalith on the abdominal X-ray inside the bladder, at first it was thought to be an ordinary bladder calculus, so a cystoscopy was performed. Even though a plain X-ray of the kidney ureter bladder has a sensitivity of $21 \%$ to $78 \%$ for detecting bladder stones, cystoscopy and computed tomography (CT) have higher sensitivity rates comparing to ultrasound or X-ray; without any study comparing cystoscopy and CT. Despite this, cystoscopy confers the advantage of detecting other possible causes of patient's symptoms, in this case discovering a fecalith adherent to the bladder's wall [9].

There are different methods of how an imaging study can be accomplished; these include CT scan, magnetic resonance imaging (MRI), retrograde cystogram, cystoscopy, and barium enema. According to these assessments, the probability of the right diagnosis is still low. Haas et al. [7] in their review of 100 patients with AVF, the diagnosis was made only in $37 \%$ on cystograms and $43 \%$ on barium enema. The diagnostic accurateness of cystoscopy is reported to be $40 \%$. In a case report of an appendico-ileo-vesical fistula, the CT and MRI failed to reveal whether the bowel adjacent to the inflammatory mass was perforated or only adherent [10]. Furthermore, colonoscopy has been widely used to investigate possible cases of colovesical fistula, but false-negative results are very frequent. This tool is also useful to exclude other intestinal pathologies [11].

Males between 10 and 40 years are more frequently affected than females, with a ratio of 4:1 [7]. This predominance is because, in the female, the uterus forms a barrier between the bladder and bowel so that communication between these organs is anatomically more difficult [4]. Our patient was also in this same age group.

The most common and consistent symptom is recurrent UTI. The symptoms are usually vague, and diagnosis is hence delayed $[12,13]$. Other symptoms and signs, which are less frequently encountered and are nonspecific, included: abdominal pain, dysuria, frequency, pyuria, bladder tenesmus, hematuria, fever, vomiting, and nausea $[3,7]$. Our patient presented lower abdominal pain, dysuria, and the impossibility to start urination, mimicking a ureterolithiasis.

Because acute appendicitis is the most common disease in the acute abdomen [14], we supposed our patient had an episode of acute appendicitis without realizing it; nevertheless, he doesn't remember having any severe abdominal pain. We contemplate our patient's appendix was in a pelvic position when he had the appendicitis episode, and the inflamed serosa of the vermiform appendix got attached to the bladder and penetrated its wall, establishing a fistulous communication.

Surgical procedures are appendectomy, excision of the fistula tract, and bladder wall repair with partial cystectomy [15]. Conventional or laparoscopic surgical approaches can be used for treatment. The former has been used for diagnosis and treatment for many years; recently, the latter has also been used with excellent outcomes in 6 patients up to the present according to our literature review [3]. Recently, Kibar et al. [11] published a case of a 29 year-old-male with AVF who was successfully treated by a robot-assisted laparoscopic approach. In our case, we decided to perform a midline incision for getting access to the bladder dome; we auspiciously removed the fistula and the portion of the bladder where the appendix was fistulized and did a primary repair of it-followed by catheter drainage and appropriate antibiotics to clear up the UTI. The patient's course was uneventful and was discharged home on POD 5.

The marked similarities between our case, and the cases reported in the literature, should be emphasized because, they reflect the persistent difficulties in establishing the diagnosis of AVF, despite the advancement in medical science, since the first reported case, making the diagnosis a real challenge for the clinician. When encountering an opening with feces or mucus in the bladder wall while performing a cystoscopy, an enterovesical fistula should be suspected, and a laparoscopic or open approach should be carried out.

\section{CONFLICTS OF INTEREST}

No potential conflict of interest relevant to this article was reported.

\section{REFERENCES}

1. Keen WW. A case of appendicitis in which the appendix became permanently soldered to the bladder, like a third ureter producing a urinary fecal fistula. Trans Am Surg Assoc 1898;16:243-52.

2. Trinavarat $\mathrm{P}$, Mahayosnond A, Riccabona M. Appendico-vesical fistula: a clinical and imaging challenge-a case report based discussion of imaging implication. Eur J Radiol Extra 2009;71:e1013.

3. García-Muñoz-Najar A, Carrión-Álvarez L, Medina-García M, García-González MD, Pereira-Pérez F. Appendicovesical fistula treated with elective laparoscopic surgery. Cir Cir 2013;81:344-7.

4. Abubakar AM, Pindiga UH, Chinda JY, Nggada HA. Appendicovesical fistula associated with Hirschsprung's disease. Pediatr Surg Int 2006;22:617-8.

5. Athanassopoulos A, Speakman MJ. Appendicovesical fistula. Int Urol Nephrol 1995;27:705-8.

6. Alis D, Samanci C, Namdar Y, Ustabasioglu FE, Yamac E, Tutar O, et al. A very rare complication of acute appendicitis: appendicovesical fistula. Case Rep Urol 2016;2016:4517029.

7. Haas GP, Shumaker BP, Haas PA. Appendicovesical fistula. Urology 1984;24:604-9.

8. Cakmak MA, Aaronson IA. Appendicovesical fistula in a girl with cystic fibrosis. J Pediatr Surg 1997;32:1793-4.

9. Türk C, Donaldson JF, Neisius A, Petrik A, Seitz C, Skolarikos A, et al. EAU Guidelines: bladder stones. Arnhem, the Netherlands: 


\section{Coloproctology Mauricio Gonzalez-Urquijo, et al.}

EAU Guidelines Office; 2020 [cited 2020 Jun 17]. Available from: https://uroweb.org/guideline/bladder-stones/\#3.

10. Kawamura YJ, Sugamata Y, Yoshino K, Abo Y, Nara S, Sumita T, et al. Appendico-ileo-vesical fistula. J Gastroenterol 1998;33:86871.

11. Kibar Y, Yalcin S, Kopru B, Kaya E, Topuz B, Ebiloglu T. Robotassisted laparoscopic repair of spontaneous appendicovesical fistula. J Endourol Case Rep 2016;2:111-3.

12. Keane S, Tebala GD. Appendicovesical fistula presenting as hypokalaemic hyperchloraemic metabolic acidosis: a case report. Ann
R Coll Surg Engl 2019;101:e131-2.

13. Sharifiaghdas F, Ghaed M, Mirzaei M. Appendico-vesical fistula in a woman. Urol J 2014;11:1703-5.

14. Lai CW, Chen JH, Wu MH, Chen CW. Complicated appendicitis with unusual complication: appendicovesical fistula. Am J Med Sci 2015;349:376-7.

15. Chung CW, Kim KA, Chung JS, Park DS, Hong JY, Hong YK. Laparoscopic treatment of appendicovesical fistula. Yonsei Med J 2010;51:463-5. 